\title{
ON THE STRUCTURE OF CERTAIN BOUNDED LINEAR OPERATORS ${ }^{1}$
}

\author{
G. D. ALLEN
}

ABSTRACT. If every function $f$ in the range of a bounded linear operator on $L_{p}$ is equal to zero on a set of measure greater than a fixed number $\epsilon$, it is shown that there is a common set of measure $\epsilon$ on which every function is zero. A decomposition theorem for such operators is proved.

1. Introduction. Consider the separable $L_{p}$ space, $p \geq 1$, with finite measure denoted by $|\cdot|$. Throughout this paper all operators $T$ are bounded linear operators mapping $L_{p}$ into itself. Define for $f \in L_{p}$ the set $K(f) \equiv$ $\{x \mid(T f)(x)=0\}$. We will establish that if $|K(f)| \geq \epsilon>0$ for all $f \epsilon L_{p}$, then there is a set $K$ with $|K| \geq \epsilon$ for which $|K \cap K(f)| \geq \epsilon$ for all $f \in L_{p}$.

The problem of considering the sets $K(f)$ arises naturally when $L_{p}$ is defined over a probability space $(\Omega, B, P)$. In this setting the sets $K(f)$ are events and the result establishes the existence of an event on which the range of $T, R(T)$, is zero almost everywhere.

2. Main results and applications. The principal result we wish to establish is the content of

Theorem 1. If $T$ is a bounded linear operator on $L_{p}$, and if $|K(f)| \geq \epsilon$ $>0$ for all $f \in L_{p}$, then there is a set $K$ with $|K| \geq \epsilon$ such that $|K \cap K(f)|$ $\geq \epsilon$ for all $f \in L_{p}$,

Proof Let $f_{1}$ and $f_{2}$ be in $L_{p}$. Define $f_{\alpha}=\alpha f_{1}+f_{2}$. By hypothesis $\left|K\left(f_{\alpha}\right)\right| \geq \epsilon$. Each of the sets $K\left(f_{a}\right)$ can be decomposed into two disjoint components, one consisting of $K\left(f_{1}\right) \cap K\left(f_{2}\right)$, and the other being the set $M\left(f_{\alpha}\right) \equiv\left\{x \mid \alpha\left(T f_{1}\right)(x)=-\left(T f_{2}\right)(x) \neq 0\right\}$, for $\alpha \neq 0$. The sets $M_{\alpha}$ are mutually exclusive and $\left.\Sigma_{\alpha \in(-\infty, \infty)}\left|M_{\alpha}\right|\right)<\infty$. Thus, at most a countable number of the sets $M\left(f_{\alpha}\right)$ have positive measure. Let $\alpha^{*}$ be a number not in this countable set. Then $K\left(f_{a^{*}}\right)=K\left(f_{1}\right) \cap K\left(f_{2}\right) \cup M\left(f_{a^{*}}\right)$. Since $\left|M\left(f_{a^{*}}\right)\right|=0$, we must have $\epsilon \leq\left|K\left(f_{a^{*}}\right)\right|=\left|K\left(f_{1}\right) \cap K\left(f_{2}\right)\right|$. Thus, the result is established for the linear subspace of $L_{p}$ spanned by $f_{1}$ and $f_{2}$.

Now define the function $f_{a \beta}=\alpha f_{1}+f_{2}+\beta f_{3}$. As above, each $K\left(f_{\alpha \beta}\right)$ can be decomposed into two disjoint components,

Received by the editors December 11, 1974 .

AMS (MOS) subject classifications (1970). Primary 47A35; Secondary 60G99.

1 This work was supported in part by the National Science Foundation under Grant GP-38486. 


$$
M\left(f_{a \beta}\right)=\left\{x \mid\left(\alpha\left(T f_{1}\right)+\left(T f_{2}\right)\right)(x)=-\beta\left(T f_{3}\right)(x) \neq 0\right\}
$$

and $K\left(f_{\alpha}\right) \cap K\left(f_{3}\right)$. Thus there is a $\beta^{*}=\beta^{*}(\alpha)$ so that $\left|M\left(f_{\alpha} \beta^{*}\right)\right|=0$, and $\epsilon \leq\left|K\left(f_{\alpha \beta^{*}}\right)\right|=\left|K\left(f_{\alpha}\right) \cap K\left(f_{3}\right)\right|$. Taking $\alpha=\alpha^{*}$ we have that $\epsilon \leq\left|K\left(f_{\alpha^{*} \beta^{*}}\right)\right|=$ $\left|K\left(f_{1}\right) \cap K\left(f_{2}\right) \cap K\left(f_{3}\right)\right|$. Proceeding by induction we conclude that $\left|\bigcap_{j=1}^{n} K\left(f_{j}\right)\right| \geq \epsilon$ for every set $\left\{f_{1}, \ldots, f_{n}\right\} \subseteq L_{p}$. Now let $f_{1}, f_{2}, \ldots$ be a basis for $L_{p}$. By the monotone convergence theorem, $\left|\bigcap_{j=1}^{\infty} K\left(f_{j}\right)\right| \geq \epsilon$. Define $K=\bigcap_{j=1}^{\infty} K\left(f_{j}\right)$. Any $f \in L_{p}$ has the representation $f=\Sigma a_{i} f_{i}$ and, since $T$ is bounded, $T f=\Sigma a_{i} T f_{i}$. So $K(f) \supseteq K$, and $|K \cap K(f)| \geq \epsilon>0$, and the theorem is proved.

Corollary 1. Let $T$ satisfy the hypotheses of Theorem 1, with $\epsilon$ chosen as large as possible. Then $T=\chi_{K} c^{T}{ }_{1}$, where $T_{1}$ is a bounded linear operator on $L_{p}$ for which there exists a $f \in L_{p}$ such that $\left|\left\{x \mid\left(T_{1} f\right)(x)=0\right\}\right|=0$. ( $K^{C}$ is the complement of $K$. )

Proof. Let $K$ be the set constructed in Theorem 1. If we define $T_{1}=$ $\chi_{K}+T$ it is clear that for every $\delta>0$ there is an $f \in L_{p}$ such that $\left|\left\{x \mid\left(T_{1} f\right)(x)=0\right\}\right|<\delta$. Suppose now that the following condition holds:

$$
\forall f \in L_{p}, \quad\left|K_{1}(f)\right|=\left|\left\{x \mid\left(T_{1} f\right)(x)=0\right\}\right|>0 .
$$

Define $S_{n}$ to be the subset of $L_{p}$ consisting of $f$ such that $\left|K_{1}(f)\right| \geq(1 / n)$. A simple argument shows that $S_{n}$ is closed. By $(*), \bigcup_{n} S_{n}=L_{p}$; therefore, some $S_{n_{0}}$ contains an open ball with, say, center $f_{0}$. Because $T$ is continuous, we can assert the existence of a smaller ball of radius $\delta^{\prime}$ and center $f_{0}$ such that if $\left\|f-f_{0}\right\|_{p}<\delta^{\prime}$, then $\left|K_{1}(f) \cap K_{1}\left(f_{0}\right)\right| \geq\left(1 / 3 n_{0}\right)>0$. For it is easy to see that

$$
\|T\|\left\|f-f_{0}\right\|_{p} \geq\left\|T f-T f_{0}\right\|_{p} \geq\left(\int_{M}\left|\left(T f_{0}\right)(x)\right|^{p} d x\right)^{1 / p},
$$

where $M=K_{1}(f)-\left(K_{1}(f) \cap K_{1}\left(f_{0}\right)\right)$. Unless $\left|K_{1}(f) \cap K_{1}\left(f_{0}\right)\right|$ is large enough, the last integral above will exceed $\delta^{\prime}\|T\|$. Thus, some $S_{n_{1}}$ contains a ball about the origin and, hence, $S_{n_{1}}=L_{p}$.

From the proof of Theorem 1, we obtain

Corollary 2. If $T_{1}$ is a bounded linear operator on $L_{p}$ of a finite measure space, and if $T_{1}$ satisfies $(*)$, then for every $f_{1}, f_{2} \in L_{p}$ there is a constant $\alpha$, as small as desired such that $\left|K_{1}\left(f_{1}+\alpha f_{2}\right)\right|=\left|K\left(f_{1}\right) \cap K\left(f_{2}\right)\right|$.

For an elementary application of Theorem 1, we remark that if two second order Gaussian stochastic processes $x_{1}(t)$ and $x_{2}(t)$ are related by a bounded linear operator $T$, that is, $x_{1}(t)=T x_{2}(t)$ for each $t$, if $T$ commutes with the resolution of identity induced by $x_{2}(t)$, and if $T$ satisfies the conditions of Theorem 1 , then $x_{1}(t)$ and $x_{2}(t)$ cannot have the same spectral type. (Cf. Hida [1] for the appropriate definitions of spectral type.) 
Acknowledgement. The author would like to thank the referee who supplied the proof of Corollary 1.

\section{REFERENCES}

1. T. Hida, Canonical representations of Gaussian processes and their applications, Mem. Coll. Sci. Univ. Kyoto Ser. A Math. 33 (1960/61), 109-155. MR 22 \#10012.

DEPARTMENT OF MATHEMATICS, TEXAS A \& M UNIVERSITY, COLLEGE STATION, TEXAS 77843 